\title{
Electroencephalography in systemic lupus erythematosus patients with neuropsychiatric manifestations
}

\author{
Howaida E. Mansour ${ }^{1}$, Reem A. Habeeb², Noran O. El-Azizi ${ }^{1}$, Heba H. Afeefy ${ }^{2}$, Marwa A. Nassef ${ }^{3}$, \\ Ahmed A. Abd Alkader ${ }^{1}$ and Naglaa Afifi ${ }^{{ }^{*}}$ (i)
}

\begin{abstract}
Background: Neuropsychiatric manifestations are frequently reported in systemic lupus erythematosus (SLE) patients. This study was done to describe electroencephalographic (EEG) findings in SLE patients with neuropsychiatric manifestation (NPSLE).

Results: Among 60 SLE patients, there were 50 females (83.3\%) and 10 males (16.7\%). EEG abnormalities were reported in 12 patients out of $30(40 \%)$ with NPSLE, while all patients with non-NPSLE $(n=30)$ had no EEG abnormalities; diffuse slowing (20\%) was the most common abnormalities, followed by generalized epileptiform activity (13.3\%), and lastly temporal epileptiform activity (6.7\%). Seizure was the most reported neuropsychiatric disorder in 13 patients (43.3\%); 8 of them had abnormal EEG (61.5\%). Periventricular white matter lesion (23.3\%) followed by infarction (13.3\%) were the most common MRI brain findings among 53.3\% of NPSLE group. Half of the cases with EEG abnormality had normal brain MRI. SLEDAI score and ACL IgM positivity were higher in the NPSLE group than the non-NPSLE group. EEG is not a sensitive or specific test for detecting NPSLE with sensitivity (37.5\%) and specificity (57.1\%).

Conclusion: Not all patients with NPSLE must have abnormal brain MRI or EEG. EEG is a useful assistant tool in the assessment of different manifestations of NPSLE, but it cannot be used as a screening test alone and must be supplemented by neuroimaging studies.
\end{abstract}

Keywords: Electroencephalography, Systemic lupus erythematosus, Neuropsychiatric manifestations

\section{Background}

SLE is a prototypic autoimmune disease characterized by the production of autoantibodies against cell nucleus giving rise to diverse clinical manifestations that almost affect all organ systems [1]. Central nervous system involvement in SLE is a characteristic of severe disease [2] as it drives most of the SLE disease morbidity and mortality [3].

Nervous system involvement in SLE leads to a heterogeneous group of neurological and psychiatric (neuropsychiatric) symptoms. Any of these neuropsychiatric

\footnotetext{
*Correspondence: dr.naglaaafifi@gmail.com

'Department of Internal Medicine and Rheumatology, Faculty of Medicine, Ain Shams University, Cairo, Egypt

Full list of author information is available at the end of the article
}

(NP) events can be directly attributed to SLE (NPSLE) or to an alternative etiology [4].

NPSLE is frequently reported in $75 \%$ of SLE patients and that varied from mild presentation like headache or mood disturbance to life-threatening conditions as with acute confusional state, major fits, stroke, or transverse myelitis [5]. The wide range of presentations and differential diagnosis often pose a difficult diagnostic challenge for clinicians [6].

Multiple mechanisms have been implicated in playing a role in the pathogenesis of NPSLE, including various arms of the immune system as well as non-immune and environmental factors that could cause blood-brain barrier (BBB) dysfunction [7]. 
Electroencephalography (EEG) is central to the diagnosis and management of epileptic disorders, but it can also help in the diagnosis and prognosis of diffuse encephalopathies, organic brain syndromes, and dementias. EEG can play an important discriminative role in several cases [8].

Routine and quantitative EEG was used to determine whether there is a lateralized pattern of electrophysiologic dysfunction in SLE patients with diverse neuropsychiatric manifestations or not. Epileptiform discharges were observed in some SLE patients. These EEG abnormalities were mostly observed in patients with clinical NPSLE and that abnormal EEG may be an indicator for subclinical NPSLE [1].

Our aim was to describe electroencephalographic findings in a cohort of SLE patients with different neuropsychiatric manifestations (NPSLE), its correlation with the disease activity parameters, and comparing them to SLE patients without neuropsychiatric manifestations (non-NP SLE).

\section{Methods}

It was a cross-sectional, observational study involving 60 adult patients diagnosed with SLE according to the 2015 ACR/SLICC Classification Criteria for SLE [9]. The patients were recruited from the Rheumatology Outpatient Clinic and Internal Medicine and Rheumatology Department. Based on the ACR criteria for neuropsychiatric manifestations [10], patients were classified into 2 groups: 30 patients with neuropsychiatric manifestations (NPSLE) as cases and 30 patients without neuropsychiatric manifestations (non-NPSLE) as controls. SLE patients with end-stage renal disease, any metabolic disorders, or drug-related side effects were excluded from the study.

All patients were subjected to the following: complete history taking including neuropsychiatric manifestations, full clinical examination including rheumatological and neurological examinations, and assessment of SLE disease activity using the SLE disease activity index (SLEDAI) score [11]. Laboratory investigations including complete blood count $(\mathrm{CBC})$, erythrocyte sedimentation rate (ESR), C-reactive protein (CRP), blood urea nitrogen (BUN), serum creatinine, urine analysis, protein/creatinine $(\mathrm{P} / \mathrm{C})$ ratio, complement $\mathrm{C} 3, \mathrm{C} 4$, lupus anticoagulant (LAC), and anticardiolipin (ACL) antibodies IgM and IgG and EEG were done for all participants at the neurology department, using Nihon Kohden LS-120 device. MRI brain was done for patients with NPSLE.

\section{Ethics approval and consent to participate}

The study was approved by the research ethical committee, faculty of medicine, FWA000017585 (No: FMASU R 13/2017). All patients included in this study gave an informed written consent to participate in this research.

\section{Statistical methods}

The collected data were coded, tabulated, and statistically analyzed using the SPSS program (Statistical Package for Social Sciences) software version 24. The description of quantitative variables is as mean, standard deviation (SD), and range. The description of qualitative variables is as number (no) and percentage (\%). Chisquare test was used to compare qualitative variables. Fisher's exact test was used instead of chi-square test when there is one expected cell or more $\leq 5$. Comparison between two independent mean groups was done using Student's $t$ test for parametric data and MannWhitney test for nonparametric data. The $P$ value was considered: $P>0.05=$ insignificant, $P<0.05=$ significant, $P<0.001=$ highly significant. Diagnostic validity of EEG was assessed and include the following: (a) the diagnostic sensitivity: it is the percentage of diseased cases truly diagnosed (TP) among total diseased cases (TP + FN); (b) the diagnostic specificity: it is the percentage of non-diseased truly excluded by the test (TN) among total non-diseased cases (TN + FP); (c) the predictive value for a + Ve test (PPV): it is the percentage of cases truly diagnosed among total positive cases; (d) the predictive value for a -Ve test (NPV): it is the percentage of cases truly negative among total negative cases; and (e) the diagnostic accuracy of the test: it is the percentage of cases truly diseased plus truly non-diseased among total cases.

\section{Results}

Among 60 SLE patients, there were 50 females (83.3\%) and 10 males (16.7\%); the patients were classified into 2 groups: (1) group I-30 patients without neuropsychiatric manifestations (non-NPSLE), and (2) group II-30 patients with neuropsychiatric manifestations (NPSLE).

In group I, there were 26 females $(86.7 \%)$ and 4 males $(13.3 \%)$, and their age ranged from 16 to 44 years, with a mean \pm SD of $25.97 \pm 7.39$ years, while in the group II, there were 24 females (80\%) and 6 males (20\%), and their age ranged from 15 to 55 years, with a mean \pm SD of $30.87 \pm 9.61$. There was a statistically significant difference between the two groups as regards the mean of age being more in group II than group I $(P<0.05)$.

Mainly 6 neuropsychiatric manifestations were reported in cases of group II. The commonest was seizure disorders (43.3\%), followed by psychosis (20\%), cerebrovascular disease (stroke syndromes and sinus thrombosis) (16.7\%), acute confusional state (13.3\%), headache (10\%), and lastly, demyelinating syndrome (6.7\%) (Fig. 1).

There was a statistically significant difference between the two groups as regards the frequency of the ACL IgM positivity and the SLEDAI score being higher in group II than group I; there were twenty three patients that had mild and moderate disease activity (SLEDAI $<10$ ), and 6 


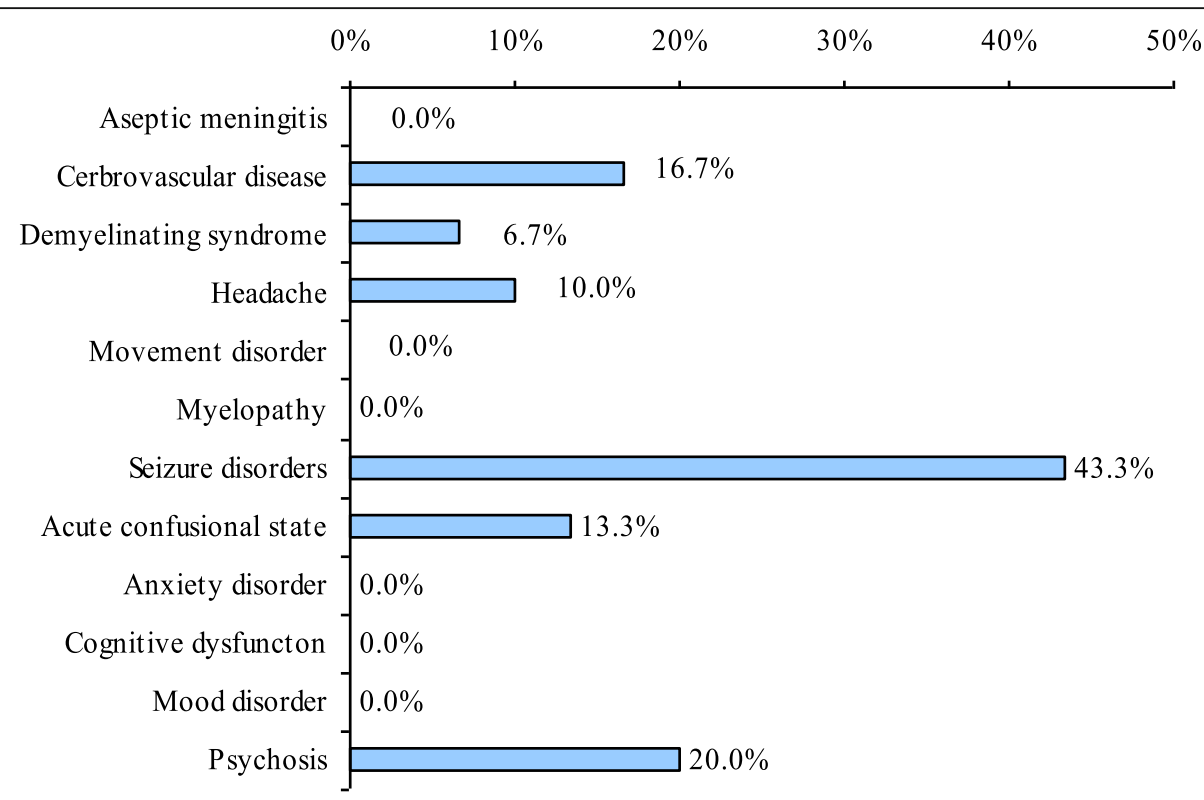

Fig. 1 Neuropsychiatric manifestations in the case group (group II)

of them had NPSLE, 15 patients had severe disease activity (SLEDAI 11-19), 13 of them had NPSLE, and 14 patients had very severe disease (SLEDAI > 20) with 11 of them that had NPSLE and no difference regarding treatment modalities (Table 1).
While no EEG finding was reported in any of the group I patients, 12 patients (40\%) of group II had abnormal EEG. The most common finding was diffuse slowing in 6 patients (20\%), followed by generalized epileptiform activity in 4 patients (13.3\%), and lastly,

Table 1 Comparison between group I and group II as regards autoimmune markers, treatment, and SLEDAI score

\begin{tabular}{|c|c|c|c|c|}
\hline & Group I, no. (\%) & Group II, no. (\%) & $\boldsymbol{P}$ value & Sig. \\
\hline Consumed C3 & $11(36.7 \%)$ & $11(36.7 \%)$ & 1.000 & NS \\
\hline Consumed C4 & $11(36.7 \%)$ & $11(36.7 \%)$ & 1.000 & NS \\
\hline LAC & $7(23.3 \%)$ & $14(46.7 \%)$ & 0.058 & NS \\
\hline ACL IgG & $6(20 \%)$ & $13(43.3 \%)$ & 0.052 & NS \\
\hline$A C L \lg M$ & $5(16.7 \%)$ & $12(40 \%)$ & 0.045 & $\mathrm{~S}$ \\
\hline Steroids & $8(26.7)$ & 9) 30.0( & 0.774 & NS \\
\hline Hydroxychloroquin & $8(26.7)$ & 14 )46.7( & 0.108 & NS \\
\hline Azathioprine & $16(53.3)$ & 15 )50.0( & 0.796 & NS \\
\hline Cyclophosphamide & $23(76.7)$ & $23) 76.7$ & 1.000 & NS \\
\hline MMF & $25(83.3)$ & 29 )96.7( & 0.085 & NS \\
\hline $\begin{array}{l}\text { SLEDAI } \\
\text { Median (IQR) } \\
\text { Range }\end{array}$ & $4(0-8)$ & $16(12-24)$ & 0.000 & HS \\
\hline No activity (0) & $0-28$ & $8-38$ & 0.002 & HS \\
\hline Mild activity (1-5) & $8(26.7 \%)$ & $0(0.0 \%)$ & 0.001 & HS \\
\hline Moderate activity (6-10) & $9(30.0 \%)$ & $0(0.0 \%)$ & 0.542 & NS \\
\hline High activity (11-19) & $8(26.7 \%)$ & $6(20.0 \%)$ & 0.001 & HS \\
\hline Very high activity $(\geq 20)$ & $2(6.7 \%)$ & $13(43.3 \%)$ & 0.015 & $S$ \\
\hline
\end{tabular}

$P$ value $>0.05$ : nonsignificant $(\mathrm{NS}) ; P$ value $<0.05$ : significant $(\mathrm{S}) ; P$ value $<0.01$ : highly significant $(\mathrm{HS})$

C3, C4 complement 3 and 4, LAC lupus anticoagulant, $A C L$ anticardiolipin, MMF mycophenolate mofetil, SLEDAI SLE disease activity using the SLE disease activity index 
Table 2 EEG findings in group II

\begin{tabular}{|c|c|c|c|c|}
\hline & & & Grou & \\
\hline & & & No. & $\%$ \\
\hline EEG & & Negative & 18 & $60 \%$ \\
\hline & & Positive & 12 & $40 \%$ \\
\hline Diffuse slowing & & Positive & 6 & $20 \%$ \\
\hline Generalized epileptiform a & & Positive & 4 & $13.3 \%$ \\
\hline Temporal epileptiform acti & & Positive & 2 & $6.7 \%$ \\
\hline Neuropsychiatric manifest & & EEG findi & & \\
\hline Seizure & $13(43.3 \%)$ & $8(61.5 \%)$ & $2(15$ & \\
\hline & & & $4(30$ & hactivity \\
\hline & & & $2(15$ & ctivity \\
\hline Headache & $3(10.0 \%)$ & 0 & - & \\
\hline Cerebrovascular disease & $5(16.7 \%)$ & 0 & - & \\
\hline Demyelinating syndrome & $2(6.7 \%)$ & 0 & - & \\
\hline Acute confusional state & $4(13.4 \%)$ & $4(100 \%)$ & $4(10$ & \\
\hline Psychosis & 6 (20.0\%) & 1 (16.7\%) & Diffu & \\
\hline
\end{tabular}

EEG electroencephalography

temporal epileptiform activity in 2 patients (6.7\%). Out of 30 patients with NPSLE, 13 patients had seizure disorders (43.3\%), 8 of them had abnormal EEG (61.5\%), 4 patients $(30.8 \%)$ of them had generalized epileptiform activity, 2 patients (15.4\%) had diffuse slowing, and 2 patients (15.4\%) had temporal epileptiform activity in EEG. Diffuse slowing EEG finding was reported among all 4 cases of acute confusional state and one of those presented by psychosis. No EEG abnormality was found among cases with headache, demyelinating syndrome, and cerebrovascular disease (Table 2).
MRI brain findings were reported in 16 patients (53.3\%) of group II (Fig. 2). The most common finding was periventricular white matter lesion in 7 patients (23.3\%), followed by infarction in 4 patients $(13.3 \%)$, subcortical white matter lesion in 2 patients $(6.7 \%)$, and demyelinating lesion in 2 patients (6.7\%). Sinus thrombosis, cerebral edema, and encephalomalacia were reported only one time (3.3\%) (Fig. 2). Among cases with EEG abnormality, MRI findings were reported in 6 cases (50\%), and all of them had periventricular white matter lesion and subcortical white matter lesions in one case.

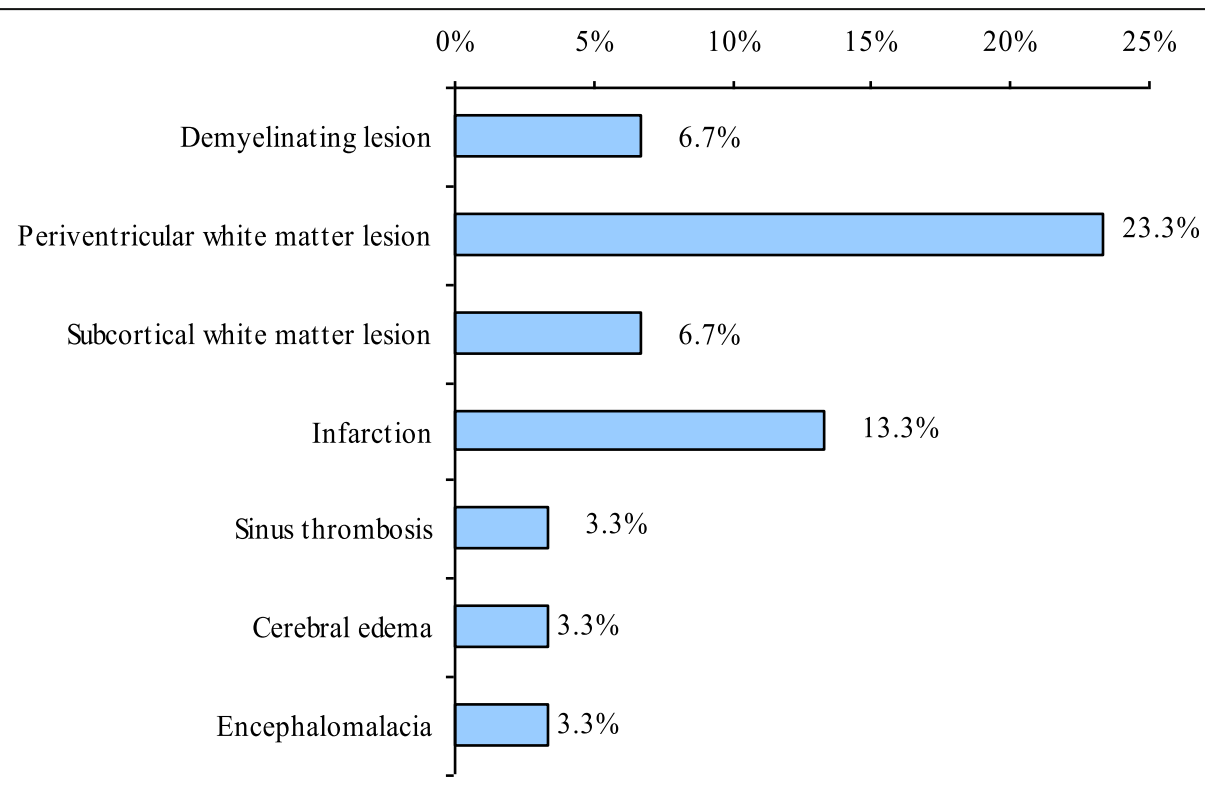

Fig. 2 MRI findings in NPSLE group (group II) 
Table 3 Comparison between group lla and group Ilb as regard age, sex, and drug history; SLEDAl; and MRI brain

\begin{tabular}{|c|c|c|c|c|c|}
\hline & & Group Ila & Group IIb & $\boldsymbol{P}$ value & Sig. \\
\hline & & No. $=18$ & No. $=12$ & & \\
\hline \multirow[t]{2}{*}{ Age } & Mean \pm SD & $31.11 \pm 11.36$ & $30.50 \pm 6.60$ & 0.868 & NS \\
\hline & Range & $15-55$ & $20-42$ & & \\
\hline \multirow[t]{2}{*}{ Sex } & Female & $16(88.9 \%$ & $8(66.7 \%)$ & 0.136 & NS \\
\hline & Male & $2(11.1 \%)$ & $4(33.3 \%)$ & & \\
\hline Steroids & Positive & $13(72.2 \%)$ & $8(66.7 \%)$ & 0.745 & NS \\
\hline \multirow[t]{2}{*}{ Steroid dose/day } & Median (IQR) & $15(10-30)$ & $17.5(10-30)$ & 0.749 & NS \\
\hline & Range & $5-30$ & $5-30$ & & \\
\hline Hydroxychloroquine & Positive & $9(50.0 \%)$ & 7 (58.3\%) & 0.654 & NS \\
\hline Azathioprine & Positive & $9(50.0 \%)$ & $6(50.0 \%)$ & 1.000 & NS \\
\hline Cyclophosphamide & Positive & $5(27.8 \%)$ & $2(16.7 \%)$ & 0.481 & NS \\
\hline MMF & Positive & $0(0.0 \%)$ & $1(8.3 \%)$ & 0.213 & NS \\
\hline \multirow[t]{2}{*}{ SLEDAI } & Median (IQR) & $15(10-24)$ & $19(12-25.5)$ & 0.396 & NS \\
\hline & Range & $8-38$ & $8-34$ & & \\
\hline MRI brain & Positive & $10(55.6 \%)$ & $6(50.0 \%)$ & 0.765 & NS \\
\hline
\end{tabular}

$P$ value $>0.05$ : non-significant (NS), $P$ value $<0.05$ : significant $(S), P$ value $<0.01$ : highly significant (HS)

$M M F$ mycophenolate mofetil, SLEDAI SLE disease activity using the SLE disease activity index, MRI magnetic resonance imaging

The 30 patients of group II (cases) were further subdivided according to EEG findings into 2 groups: (1) group IIa with negative EEG (18 patients), and (2) group IIb with positive EEG (12 patients).

There was no statistically significant difference $(P>$ 0.05 ) between the two groups as regard age, sex, and drug history. There was no statistically significant difference $(P>0.05)$ between the two groups neither for SLEDAI score nor MRI abnormality (Table 3). EEG was not sensitive nor specific for detecting NPSLE compared to the brain MRI with sensitivity (37.5\%), specificity (57.1\%), PPV (50.0\%), and NPV (44.4\%), and the overall test accuracy was $46.7 \%$ (Table 4 ).

\section{Discussion}

Neuropsychiatric systemic lupus erythematosus (NPSLE) is one of the serious and the least understood of lupus presentations that have various multifactorial pathogenesis [12].

The American College of Rheumatology in 1999 defines NPSLE in a list of 19 neurological manifestations, with 12 of them involving the central nervous system and 7 peripheral nervous system manifestation. The CNS manifestations were further sub-divided into focal and diffuse [10].

Table 4 EEG specificity and sensitivity

\begin{tabular}{llllll}
\hline Parameter & Accuracy & Sensitivity & Specificity & PPV & NPV \\
\hline EEG & $\mathbf{4 6 . 7}$ & $\mathbf{3 7 . 5}$ & $\mathbf{5 7 . 1}$ & $\mathbf{5 0 . 0}$ & $\mathbf{4 4 . 4}$ \\
\hline
\end{tabular}

EEG electroencephalography, PPV positive predictive value, NPV negative predictive value
A variety of neuropsychiatric manifestations can occur with lupus; in the current study, mainly 6 neuropsychiatric manifestations were reported in the NPSLE group. Seizure disorder was the most frequent in 13 patients (43.3\%), followed by psychosis in 6 patients $(20 \%)$, cerebrovascular disease (stroke syndromes and sinus thrombosis) in 5 patients (16.7\%), acute confusional state in 4 patients (13.3\%), headache in 3 patients $(10.0 \%)$, and lastly, demyelinating syndrome in 2 patients (6.7\%). Unterman et al. [13] showed that the most frequent NP syndromes were headache $28.3 \%$, followed by mood disorders in $20.7 \%$ and reported seizures in $9.9 \%$. Govoni et al. [14] reported that from 326 patients with NPSLE, the most common features were headache $(26.1 \%)$, cerebrovascular events $(22.7 \%)$, mood disorders $(8.9 \%)$, seizures (14.4\%), and cognitive dysfunctions (9.5\%). In Kakati et al. [1], the nervous system was involved in 19 of 52 patients (36.54\%). The most common features were cognitive impairment (57.89\%) followed by seizure disorder (42.1\%).

In our study, there was a statistically highly significant difference between the two studied groups as regards the SLEDAI score being higher in the NPSLE group than the non-NPSLE group $(P<0.01)$. Most of NPSLE patients $(80 \%)$ had an activity score $>11$. Morrison et al. [15] reported that SLEDAI-2K scores in patients with NP events attributed to SLE were higher than in patients with NP events attributed to non-SLE causes even when $\mathrm{NP}$ variables were removed from the SLEDAI-2K. Our results were also consistent with the study done by Kakati et al. [1] in which most of the patients (46.14\%) 
had an SLEDAI score of less than 20. Almost 25\% of patients had a score between 21 and 29 .

Antiphospholipid antibodies are one of the most frequent NPSLE-associated antibodies, in a meta-analysis of 21 retrospective studies that involve more than 1000 patients with SLE and other autoimmune diseases, the average prevalence of anticardiolipin antibodies and lupus anticoagulant was 44 and 34\%, respectively. One hundred nine NP manifestations occurred in 38 to $49 \%$ of patients with antiphospholipid antibodies compared with 12 to $21 \%$ of patients without antiphospholipid antibodies, mainly were due to vascular thrombotic events such as stroke and seizures [16]. Our study showed that patients with NPSLE were significantly associated with ACL IgM positivity $(P<0.05)$. Hanly et al. [17] stated that there was a strong association between LAC and cerebrovascular disease attributed to SLE $(P=0.038)$. Our results also agreed with the results of Kakati et al. [1] in which 9 patients out of 19 in the NPSLE group had positive antiphospholipid antibodies (47.37\%) with a significant difference as compared to the non-NPSLE group (15.4\%).

Brain MRI is one of the most sensitive imaging modalities in the identification of CNS lesions in different disorders, including NPSLE [18]. About half of our NPSLE patients had normal MRI brain findings. Two previously published studies documented the brain MRI findings in large numbers of lupus populations presented by active NPSLE and found that brain MRI abnormalities were absent in almost half of the patients with active NPSLE, supporting the immune-mediated pathogenesis for such active NPSLE $[19,20]$.

Among MRI abnormalities, periventricular white matter lesion was the most frequent (23.3\%), followed by infarction (13.3\%), subcortical white matter lesion (6.7\%), and demyelinating lesion (6.7\%). Kakati et al. [1] in which MRI abnormality was found in $53.12 \%$ patients, subcortical white matter lesions were the commonest (64.7\%) followed by cortical atrophy in eight (47.0\%), and periventricular white matter lesion reported $35.2 \%$. In another study done by Glanz et al. [21], MRI was abnormal in $8 / 11$ patients (72.7\%). The most common abnormalities were atrophy, periventricular white matter changes, and subcortical white matter changes, respectively. Luyendijk et al. [22] stated that the principal findings were focal hyperintensities in white matter $(49 \%$ of all patients) or both white matter and gray matter (5\% of all patients), suggestive of vasculopathy or vasculitis.

EEG abnormalities have been described in SLE patients with diverse CNS manifestation [1, 8, 21, 23], and we reported EEG abnormality in 12 patients out of 30 (40\%) with NPSLE, while all 30 patients with nonNPSLE had no EEG abnormalities. This disagreed with the study of Kakati et al. [1], in which EEG abnormalities were found in patients with NPSLE (42.11\%) and in those with non-NPSLE (30.77\%). EEG abnormalities with diverse neuropsychiatric symptoms were identified in $14 / 20$ patients $(70 \%)$ with SLE in a study by Glanz et al. [21].

The most common EEG abnormalities in our study were diffuse slowing ( 6 patients $=20 \%$ ), followed by generalized epileptiform activity in 4 patients (13.3\%), and lastly, temporal epileptiform activity in 2 patients each $(6.7 \%)$. This was consistent with the findings reported in previous studies where slow activity was the most frequent EEG abnormalities in lupus patients [1, 21, 23].

Meanwhile, brain MRI was found to be normal among half of the patients with NP manifestations and abnormal EEG (6/12 patients), and this may indicate that EEG may show abnormalities early before the appearance of MRI lesions. And this agreed with Lampropoulos et al. [8]; they reported that half of the patients with abnormal EEG findings (19 of 37 patients [51.4\%]) had normal results of brain MRI. This finding suggests that the EEG may be more sensitive to early vascular changes than is brain MRI, which may detect more advanced stages of cerebrovascular disorder as multifocal lesions and smallvessel disease.

On another way, $55.6 \%$ of patients with normal EEG (10/18 patients) had abnormal MRI brain, which disagreed with Lampropoulos et al. [8] which stated that none of the patients with normal EEG results had abnormal findings on MRI.

The most common MRI abnormality in patients with abnormal EEG was periventricular white matter lesion. There were some differences in the study done by Kakati et al. [1], in which subcortical white matter lesions was the commonest MRI finding associated by EEG abnormality in lupus patients

Our study showed that 13 patients out of 30 with NPSLE had seizure disorders (43.3\%), and 8 of them had abnormal EEG (61.5\%). Four patients (30.8\%) out of 13 with seizure disorders had incidence of generalized epileptiform activity in EEG, 2 patients (15.4\%) had diffuse slowing, and 2 patients (15.4\%) had temporal epileptiform activity. While Glanz et al. reported that seizure disorders occurred in $19.9 \%$ of patients with SLE, EEG abnormalities were reported in $87.1 \%$ of seizure patients. In $79.6 \%$ of cases, these abnormalities predominantly affected the left hemisphere. In addition, $74.4 \%$ of patients with left hemisphere EEG abnormalities demonstrated dysfunction in the left temporal region [23].

Our study stated that EEG is not a sensitive or specific test for detecting NPSLE with sensitivity (37.5\%), specificity (57.1\%), PPV (50.0\%), NPV (44.4\%), and overall test accuracy $(46.7 \%)$. So, it is not an accurate screening test for detecting NPSLE and cannot be used alone without supplementary neuroimaging studies for diagnosis of NPSLE. The main limitations of this work were, first, the 
small sample size and, second, being a one center study and MRI done only for cases with NPSLE. We recommend further studies on larger scales and over a long duration to clarify the NPSLE predictors and different tools of evaluation with a monitor of therapeutic response.

\section{Conclusion}

Not all patients with NPSLE have abnormal EEG or brain MRI. NPSLE patients may have abnormal EEG while the MRI is still normal and the reverse is true; some NPSLE patients with abnormal MRI findings have normal EEG patterns. So, both EEG and MRI are complementary investigations in the assessment of NPSLE patients.

EEG is a useful assistant tool in the assessment of the different manifestations of NPSLE, but it cannot be used alone as a screening test for detecting NPSLE and must be supplemented by neuroimaging studies.

\section{Abbreviations}

SLE: Systemic lupus erythematosus; NPSLE: Neuropsychiatric systemic lupus erythematosus; EEG: Electroencephalography; MRI: Magnetic resonance imaging; SLEDAl: Disease activity assessment using the SLE disease activity index; LAC: Lupus anticoagulant; ACL: Anticardiolipin antibodies; CBC: Complete blood count; ESR: Erythrocytic sedimentation rate; CRP: C reactive protein; BUN: Blood urea nitrogen; P/C: Protein/creatinine ratio; C3, C4: Complement; MMF: Mycophenolate mofetil; PPV: Positive predictive value; NPV: Negative predictive value

\section{Acknowledgements}

Great thanks to all participants in this work, supervisors, and all authors for their effort in the research production.

\section{Authors' contributions}

HM: conceived the idea and planned the research work. RH: contributing the design and supervised the finding. NE: verified the analytic methods and reviewed the manuscript. MN: participated in writing the research. HA: performed part of investigations and supervised the work. AA: carried out data collection, statistical analysis, and writing. NA: contributed work planning, data analysis, and manuscript writing. All authors discussed the result and contributed to the final manuscript. The authors read and approved the final manuscript.

\section{Funding}

This work did not receive any financial support.

\section{Availability of data and materials}

Data and materials are applicable.

\section{Ethics approval and consent to participate}

The study was approved by the research ethical committee, faculty of medicine, FWA000017585 (No: FMASU R 13/2017). All patients included in this study gave an informed written consent to participate in this research.

\section{Consent for publication}

Consent for publication was taken from all participants.

\section{Competing interests}

No financial and or non-financial interests in relation to that work.

\section{Author details}

'Department of Internal Medicine and Rheumatology, Faculty of Medicine, Ain Shams University, Cairo, Egypt. ${ }^{2}$ Department of Neurology medicine, Faculty of Medicine, Ain Shams University, Cairo, Egypt. ${ }^{3}$ Department of
Physical Medicine, Rheumatology and Rehabilitation, Faculty of Medicine, Ain Shams University, Cairo, Egypt.

Received: 12 June 2020 Accepted: 27 July 2020

Published online: 01 December 2020

\section{References}

1. Kakati S, Barman B, Ahmed SU, Hussain M (2017) Neurological manifestations in systemic lupus erythematosus: a single centre study from North East India. J Clin Diagn Res 11(1):OC05-OC09

2. Hajighaemi F, Etemadifar M, Bonakdar ZS (2016) Neuropsychiatric manifestations in patients with systemic lupus erythematosus: a study from Iran. Adv Biomed Res 5:43

3. Jafri K, Patterson P, Lanata C (2017) Central nervous system manifestations of systemic lupus erythematosus. Rheum Dis Clin N Am:0889-857X/17

4. Magro-Checa C, Zirkzee EJ, Beaart-van de Voorde LJ, Middelkoop HA, van der Wee NJ, Huisman MV et al (2017) Value of multidisciplinary reassessment in attribution of neuropsychiatric events to systemic lupus erythematosus: prospective data from the Leiden NPSLE cohort. Rheumatology 56:1676-1683

5. Appenzeller S, Costallat LT, Cendes F (2006) Neurolupus. Arch Neurol 63(3): 458-460

6. Joseph FG, Lammie GA, Scolding NJ (2007) CNS lupus: a study of 41 patients. Neurology. 69(7):644-654

7. Stock AD, Wen J, Putterman C (2013) Neuropsychiatric lupus, the blood brain barrier, and the TWEAK/Fn14 Pathway. Front Immunol 4:484

8. Lampropoulos CE, Koutroumanidis M, Reynolds PP, Manidakis L, Hughes GRV, D'Cruz DP (2005) Electroencephalography in the assessment of neuropsychiatric manifestations in antiphospholipid syndrome and systemic lupus erythematosus. Arthritis Rheum 52(3):841-846

9. Salehi-Abari I (2015) 2015 ACR/SLICC revised criteria for diagnosis of systemic lupus erythematosus. Autoimmune Dis Ther Approaches 2(1):114

10. ACR Ad Hoc Committee on Neuropsychiatric Lupus Nomenclature (1999) The American College of Rheumatology nomenclature and case definitions for neuropsychiatric lupus syndromes. Arthritis Rheum 42:599-608

11. Gladman DD, Ibanez D, Urowitz MB (2002) Systemic lupus erythematosus disease activity index 2000. J Rheumatol 29:288-291

12. Popescu A, Kao AH (2011) Neuropsychiatric systemic lupus erythematosus. Curr Neuropharmacol 9(3):449-457

13. Unterman A, Nolte JE, Boaz M, Abady M, Shoenfeld Y, Zandman-Goddard G (2011) Neuropsychiatric syndromes in systemic lupus erythematosus: a meta-analysis. Semin Arthritis Rheum 41:1-11

14. Govoni M, Bombardieri S, Bortoluzzi A, Caniatti L, Casu C, Conti F et al (2012) Factors and comorbidities associated with first neuropsychiatric event in systemic lupus erythematosus: does a risk profile exist? A large multicentre retrospective crosssectional study on 959 Italian patients. Rheumatology (Oxford) 51:157-168

15. Morrison E, Carpentier S, Shaw E, Doucette S, Hanly JG (2014) Neuropsychiatric systemic lupus erythematosus: association with global disease activity. Lupus. 23:370-377

16. Love PE, Santora SA (1990) Antiphospholipid antibodies: anticardiolipin and the lupus anticoagulant in systemic lupus erythematosus (SLE) and in nonSLE disorders. Prevalence and clinical significance. Ann Intern Med 112:682698

17. Hanly JG, Urowitz MB, Sanchez-Guerrero J, Bae SC, Gordon C, Wallace DJ et al (2007) Systemic lupus international collaborating clinics, neuropsychiatric events at the time of diagnosis of systemic lupus erythematosus: an international inception cohort study. Arthritis Rheum 56: 265-273

18. Bertsias GK, loannidis JP, Aringer M, Bollen E, Bombardieri S, Bruce IN et al (2010) EULAR recommendations for the management of systemic lupus erythematosus with neuropsychiatric manifestations: report of a task force of the EULAR standing committee for clinical affairs. Ann Rheum Dis 69: 2074-2082

19. Luyendijk J, Steens SC, Ouwendijk WJ, Steup-Beekman GM, Bollen ELEM, van der Grond J et al (2011) Neuropsychiatric systemic lupus erythematosus: lessons learned from magnetic resonance imaging. Arthritis Rheum 63:722732

20. Steup-Beekman GM, Zirkzee EJ, Cohen D, Gahrmann MAB, Emmer JB, Steens CAS et al (2013) Neuropsychiatric manifestations in patients with 
systemic lupus erythematosus: epidemiology and radiology pointing to an immune-mediated cause. Ann Rheum Dis 72(Suppl 2):ii76-ii79

21. Glanz BI, Laoprasert P, Schur PH, Robertson-Thompson A, Khoshbin S (2001) Lateralized EEG findings in patients with neuropsychiatric manifestations of systemic lupus erythematosus. Clin Electroencephalogr 32(1):14-19

22. Luyendijk J, Steens SC, Ouwendijk WJ, Steup-Beekman GM, Bollen EL, van der Grond J et al (2011) Neuropsychiatric systemic lupus erythematosus: lessons learned from magnetic resonance imaging. Arthritis Rheum 63:722732

23. Glanz Bl, Schur PH, Khoshbin S (1998) EEG abnormalities in systemic lupus erythematosus. Clin Electroencephalogr 29(3):128-1331

\section{Publisher's Note}

Springer Nature remains neutral with regard to jurisdictional claims in published maps and institutional affiliations.

\section{Submit your manuscript to a SpringerOpen ${ }^{\circ}$ journal and benefit from:}

- Convenient online submission

- Rigorous peer review

- Open access: articles freely available online

High visibility within the field

- Retaining the copyright to your article

Submit your next manuscript at $\boldsymbol{\wedge}$ springeropen.com 